\begin{tabular}{|c|c|c|}
\hline Beitr. Ent. & Keltern & ISSN 0005-805X \\
\hline $51(2001) 1$ & S. $65-71$ & 14.09 .2001 \\
\hline
\end{tabular}

\title{
Klärung und Neubeschreibung der Sandbiene Andrena asiatica FRIESE, 1921
}

\section{(Hymenoptera: Apidae, Andreninae)}

Mit 3 Figuren

\author{
JOHANNES SCHUBERTH, WilHELM GRÜNWALDT und KLAUS SCHÖNITZER
}

\section{Zusammenfassung}

Andrena asiatica FRIESE, 1921 wird wiederbeschtieben und ein Lectotypus witd festgelegt. Die ron WARNCKE 1975 beschriebene, heller behaarte Unterart A. asiatica oxyura WARNCKE, 1975 ist ein Synonym der Nominatform (syn. n.). A. asiatica gehört nicht in die Untergattung Nobandrena, sondern ist vermutlich mit A. formosa Morawitz, 1878 und $A$. combusta Morawitz, 1876 verwandt.

\section{Summary}

Andrena asiatica FRIESE, 1921 is tedescribed and a lectotype is designated. The brighter haired subspecies, A. asiatica oxyura WARNCKE, 1975 is a synonym of the nominate form (syn. n.). A. asiatica does not belong to the subgenus Nobandrena but is probably related to A. formosa MORAWTTZ, 1878 and $A$. combusta MORAXITZ, 1876.

\section{Einleitung}

Andrena asiatica wurde von FRIESE im Jahre 1921 aus dem Amanausgebirge (=Alma(n) Dagh, Nur Daglari) in der heutigen Türkei beschrieben (FAHRINGER \& FRIESE, 1921). Dabei betont FrIESE die Verwandtschaft mit A. paliuri, MORAWITZ, 1887 (= A. nobilis, MORAWITZ, 1873). Auch WARNCKE (1974) stellt $A$. asiatica in die Untergattung Nobandrena, obwohl es für ihn offensichtlich keineswegs klar war, in welche Untergattung diese Art zu stellen ist: In der Arbeit von 1974 (Teil A) listet er unter der Untergattung Truncandrena eine neue Art (Andrena oxyura) auf, die er erst ein Jahr später im Teil B beschreibt, dann aber als Unterart von $A$. asiatica (Andrena asiatica oxyura WARNCKE, 1975) ${ }^{1}$. Damit stellt er oxyura zu Nobandrena (siehe auch GUSENLEITNER, 1984, S. 214) obwohl er in der Beschreibung die Ähnlichkeit mit $A$. curvungula THOMSON, 1870 hervorhebt und sie ,eine charakteristische Schuppenandrene" nennt, als gehörte sie zur Untergattung Lepidandrena.

\footnotetext{
${ }^{1}$ An diesem Beispiel zeigt es sich auch, wie wichtig es ist, sich an die Empfehlung der IRZN (Appendix B, 10: „A name should not be cited in advance of its intended establishment") zu halten (siehe auch BLANK \& KRAUS 1994; dort Wertung der ersten Nennung von A. oxyura [1974] als nomen nudum).
} 
Es fält auf, dass in der Beschreibung von $A$. asiatica oxyura (WARNCKE 1975, S. 44, Nr.23) Angaben fehlen, worin sich die neubeschriebene Unterart von der Nominatform unterscheidet, außer dass sie heller behaart ist. Bei den Männchen schreibt WARNCKE, dass sie „,nicht verschieden“ sind (es gibt allerdings in mehreren Sammlungen Männchen, die er als $A$. a. oxyura bestimmt hat). Bemerkenswert ist außerdem, dass die beiden „Unterarten" (A. a. asiatica und A. a. oxyura) nach WARNCKE (1974) im gleichen Gebiet der Türkei, zur gleichen Jahreszeit und auf den gleichen Blüten vorkommen, also scheinbar sympatrisch sind. Aus diesern Grund können es kaum echte Unterarten sein. Es stellte sich die Frage, ob es sich um zwei Arten handelt, oder ob eine der beiden Unterarten eingezogen werden muß. Nach Auffinden und Untersuchen der vollständigen Typenserie konnte diese Frage geklärt werden.

\section{Typen}

Nach der Artbeschreibung (FAHRINGER \& FRIESE 1921, S. 171f) besteht die Typenserie aus einem Weibchen und 4 Männchen. Es konnte die ganze Serie aufgefunden werden: das Weibchen und ein Männchen befinden sich im Deutschen Entomologischen Institut, Eberswalde, die beiden anderen Männchen im Museum für Naturkunde, Berlin. Aufgrund der identischen Fundortzettel ist es eindeutig, dass es sich um die Typenserie handelt, obwohl die Tiere aus Eberswalde einen Determinationszettel tragen, der mit 1926 datiert ist. Es ist anzunehmen, dass dieser erst nachträglich angebracht wurde, als die Tiere aus der gemeinsamen Sammlung genommen wurden. Dafür spricht auch, dass ein Tier aus Berlin (Lectotypus s.u.) einen größeren Determinationszettel trägt, der vermutlich als Bodenettikett diente.

Lectotypus, on, ZMB, hiermit festgelegt: „Amanusgeb. Bagtsche“ "Type“ "Andrena asiatica o" Fr. 1919 FrIESE det." „Antbrena asiatica FrIESE“" „Zool. Mus. Berlin“" „Andrena asiatica o FRIESE, 1921. LECTOTYPUS des. SCHÖNITZER 1993“ unbeschädigt, Genitalkapsel herausgezogen, auf Plättchen montiert, beim Tier. Länge $11,5 \mathrm{~mm}$. Dieses Tier wird als Lectotypus festgelegt, da es einen Typus-Zettel trägt, der vermutlich von FrIESE stammt. Auch WARNCKE hat dieses Tier als Typus bezeichnet, allerdings nicht als Lectotypus designiert (WARNCKE 1965).

Paralectotypen: 1.) ơ: „19.V. Amanusgeb. Jaribasch" Rückseite: „Campanula Bt.." (nicht ganz leserlich), „Coll. FrIESE" „Andrena asiatica o" 1919 FrIESE det. Fr." „Andrena asiatica FR. det. Dr. WARNCKE" "Zool. Mus. Berlin" „Andrena asiatica of FRIESE, 1921. PARALECTOTYPUS des. SCHÖNITZER 1993"; linkes Vorderbein fehlt; Genitalapparat herausgezogen. 2.) ơ : „Amanusgeb. Bagtsche“ „Andrena asiatica on 1919 Fr. FrIESE det." „Andrena asiatica FR. det. K. WARNCKE" „Zool. Mus. Berlin" „Andrena asiatica o" FRIESE, 1921“ „PARALECTOTYPUS des. SCHÖNITZER 1993“ Tarsus re. hinten abgebrochen. 3.) o": „Andrena asiatica o" 1926 FrIESE det.“ "Campanula Bt.."(nicht leserlich); Rückseite: „19.V. Amanusgeb. Jaribaschi“ „Coll. FrIESE det.“ Coll. DEI Eberswalde“. Genitalien herausgezogen, „PARALECTOTYPUS des. SCHÖNITZER 1993“. 4.) I: „Amanusgeb. Bagtsche" „Coll. FrIESE det." "Andrena asiatica +1926 FrIESE det." „Coll. DEI Eberswalde“ "PARALECTOTYPUS des. SCHÖNITZER 1993“. 


\section{Beschreibung}

Männchen: 11 bis $12 \mathrm{~mm}$. Kopf: Galea relativ lang, glatt mit feinen zerstreuten Punkten, nur am Rand behaart. Clypeus vor allem quer gebogen, glänzend, schwach chagriniert, punktiert (in der Medianlinie ca. 17 Punkte). An den Genae, neben dem Clypeus, ventral kleine längliche gelbe Flecken (Fig. 1), deutlich kleiner als bei den Tieren der A. nobilis-Gruppe, z. T. fehlend. Stirnschildchen rauh punktiert, Stirn rauh skulptiert, längsgerieft. Seitliche Ocellen 1 $1 \frac{2}{2}$ Ocellendurchmesser von Scheitelrand entfernt. Scapus reicht knapp bis zur Mittelocelle, stark behaart. 4. Antennenglied teilweise, die distaleren alle aufgehellt, rötlich. Form der Antennenglieder siehe Zeichnung (Fig. 1). Wangen punktiert und stark chagriniert, Breite ca. 1,3 x Augenbreite. Thorax: Pronotum chagriniert und punktiert, seitlich nicht gekielt, Rinne am Vorderrand sehr flach. Mesonotum und Scutellum chagriniert und gleichmäßig punktiert. Postscutellum rauh schuppig chagriniert, flach punktiert, Punkte schräg länglich. Mittelfeld des Mittelsegmentes rauh skulptiert mit feinen unregelmäßigen Graten. Horizontaler Teil des Propodeums grob punktiert, Seiten körnig erhaben punktiert. Metapleuren chagriniert, unpunktiert. Abdomen: Tergite deutlich chagriniert, flach punktiert. Sternite grob chagriniert und flach punktiert. Spitze des 6. Sternites tief eingekerbt, 8. Sternit ventral in der Mitte verdickt, apical in etwa rechteckig. Genitalapparat: Gonobasis leicht chagriniert, etwa 2 x so breit wie lang.
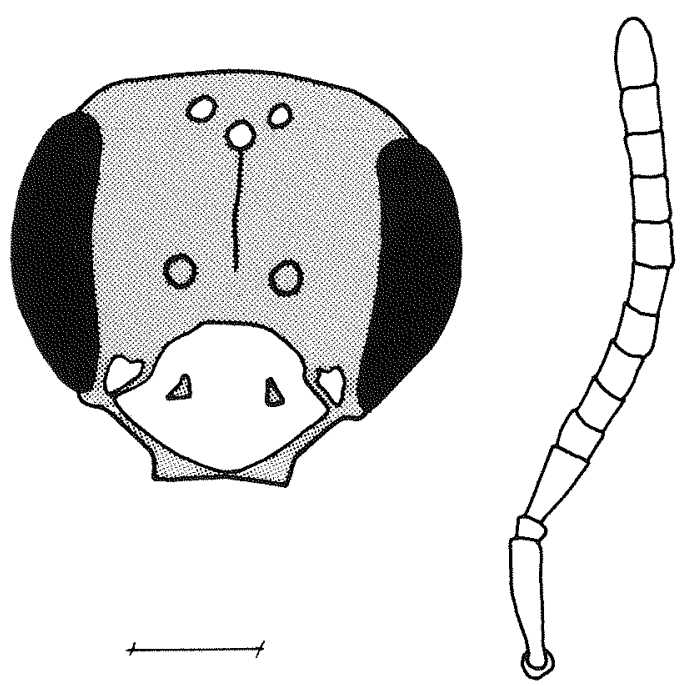

Fig. 1: A. asiatica, Männchen: Gesicht von vorne und Antenne.

Apicaler Teil des Gonostylus mit zweiseitig gefiederten Haaren, Penisvalve mit hyalinen Seitenflügeln, Spitze seitlich flach zusamengedrückt, von der Seite gesehen gleichmäßig rund (siehe Abb. 2d). Behaarung: Kopf schmutzig weiß behaart. Thorax am Rücken kurz gelblich, seitlich länger weiß behaart. Tergite locker abstehend weiß behaart, am 1 . Tergit lang, die folgenden kurz. 2. und 3. Tergit mit lockeren weißen Binden, median unterbrochen, am 4 . Tergit durchgehend; Endfranse goldbraun. Sternite schmutzig weiß behaart, an den Segmenträndern fransenartig. Achtes Sternit ventral in der Mitte stark behaart (Haare deutlich gefiedert). 
Weibchen: 12 - $14 \mathrm{~mm}$. Kopf: Gesicht vorne quadratisch. Mundwerkzeuge für die Gattung sehr lang, Galea lang, spitz, glänzend, fein punktiert. Labrumanhang breit trapezförmig. Clypeus leicht vorgezogen und allseitig gewölbt, relativ gleichmäßig fein punktiert, zwischen den Punkten chagriniert. Wange breiter als Komplexauge, fein punktiert, chagriniert. Drittes Antennenglied fast so lang wie die drei folgenden. AntennengeiBel zumindest auf der Unterseite aufgehellt. Die Foveae faciales erstrecken sich nach unten bis zur Höhe der Clypeusoberkante. Sie sind insgesamt relativ breit (knapp 0,5 x Gesichtshälfte, Fig. 2a), nach unten kaum verschmälert, fast dem Komplexauge anliegend, erstrecken sich nach oben bis zur Höhe der seitlichen Ocellen (Abstand Oberkante der Fovea/Ocelle ist weniger als 1 Ocellenbreite). Abstand der Seitenocellen vom Hinterrand des Kopfes etwa 1,5 facher Ocellendurchmesser. Thorax: Mesonotum chagriniert, dicht mit haartragenden Punkten besetzt (Fig. 2b). Mittelfeld des Mittelsegments mit in der Mitte relativ grober, an den Rändern feinerer Mikroskulpturierung. Flügel stark gebräunt, Nervulus deutlich postfurcal. Abdomen: Tergite fein chagriniert, nicht punktiert, Endränder der Tergite aufgehellt, Depressionen relativ schmal. Pygidium bei
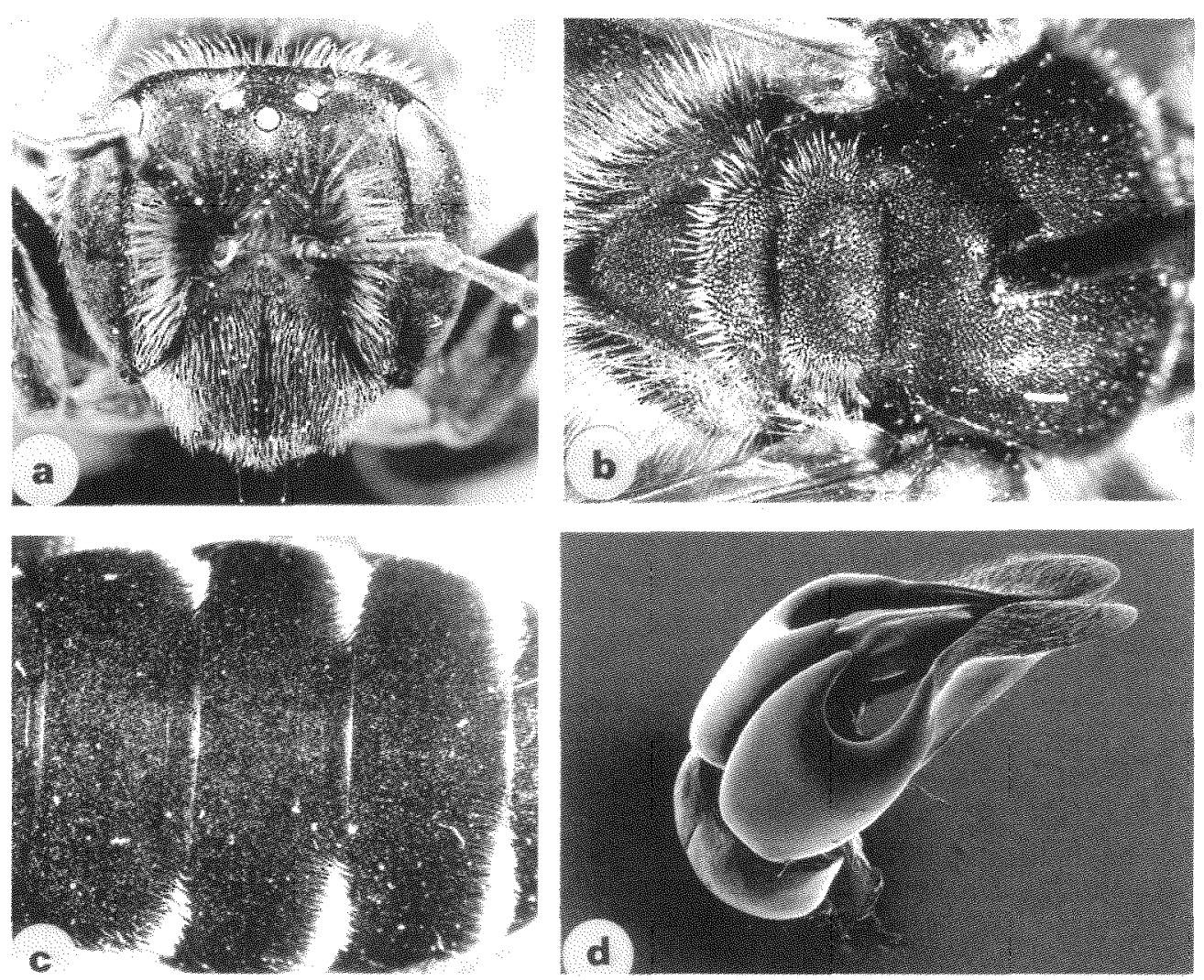

Fig. 2: A. asiatica, Gesicht des Weibchens von vorne (a), Thorax (b) und Abdomen (c) des Weibchens von dorsal. Männlicher Genitalapparat im rasterelektronenmikroskopischen Bild (d). 
manchen Tieren mit abgegrenztem Außenrand. Behaarung: Insgesamt braune mittellange Behaarung. Auffällige braune Behaarung von Mesonotum, Scutellum und Postscutellum mit kurzen spindelförmigen Haaren, die so dicht beieinander liegen, daß die darunterliegende Kutikula kaum zu etkennen ist. Körbchenboden relativ dicht behaart. Flocculus graubraun, Scopa schwach rundum gefiedert, schwarz- bis rötlichbraun. 2.-4. Tergit mit breit unterbrochener, schmaler, weißer Binde (Fig. 2c). Endfranse goldbraun bis dunkelbraun. Einzelne Tiere sind deutlich dunkler, fast schwarz behaart (siehe hierzu Diskussion).

\section{Untersuchtes Material}

Abkürzungen: DEI = Deutsches Entomologisches Institut, Eberswalde; GU = F. GUSENLEITNER, Linz; GW = W. GRÜNWALDT, München; SCH = M. SCHWARZ, Ansfelden; SW = Sammlung WARNCKE im OÖ Landesmuseum Linz; WA = leg. K. WARNCKE; ZMB = Zoologisches Museum der Humboldt Universität Berlin; ZSM = Zoologische Staatssammlung München; ZT = Zeichnung von H. TEUNISSEN, Leiden.

Türkei: Gürün: 1 ㅇ 6.3.70, SW (Holotypus von A. a. oxyura); Sivas Gürün: 2 ơ $\sigma^{\star} 2.6 .78$ leg. und coll. SCH; Şemdinli/Hakkari: $2 \sigma^{\circ}$ 12.6.81 WA; Yüksekova/Hakkari: $2 \sigma^{\circ} \sigma^{*}$ 10.6.81 WA, ZT; $60 \mathrm{~km}$ westl. Hakkari: $20^{\circ}$ 6 6.6.77 WA; Hakkari: 1 o $10^{\circ}$ 1-4.6.80 WA, $\mathrm{Sw}, 30^{7}$ 10.6.81 WA, Sw; Karakurt/Arastal: 5 우 우 $20^{7}$ o 12.6.77 WA, Sw, (1 을 ZSM); Horasan/Arastal: 1 o 14.6.73 WA, Sw; Siirt Batman: 1 o 6.6.80 SCH; Gaybi (nahe Eregli, Lycanonien): 2 ㅇ ㅇ 10.6.65 (leg. RESSL) coll. GW; Istanbul-Florya: 3 o $^{\text {o }}$ 21.5.64, leg. GW an Althaea rosea coll. GW; Muș: 5 ơ ơ 1 क 12-15.6.76 leg. F.RESSL, 1 o Sw, sonst coll. GW (1 o REM Präparat des Genitals, ZSM); Bafa See Mugla: $20^{*} o^{*}$ 20.4.81 WA, Sw; Bursa: $2 \sigma^{*}$ 25.5.64 leg. und coll. GW; Amanusgebirge (= Nur Daglari): $40^{*} 19$ 17.19.5.1914 leg. F.TÖLG (Lectotypus und Paralectotypen von $A$. asiatica, siche oben); zw. Sivas und Malatia: 1 ㅇ 6.-10.6.19 leg. MAC DANIELS Sw (Paratype von $A$. a. oxyura); Cagis vel. Bigadic: 18.4 .72 leg. R. D. DE CHENON Sw (Paratype von A. a. oxyura); Aghyr Dagh: $5 \sigma^{\star} 0^{*}$; Karaköy Sindirgi Akhisai: 18.4 .72 leg. R. D. DE CHENON Sw; Milet/Aydin: $10^{\star}$ 22.4.81 WA Sw; Kusadasi/Aydin: $20^{\star} o^{\star}$ 19.4.81 WA Sw. - Samos: Kukkari, $10^{*}$ 2.5.77 leg. Teunissen, ZT. - Lesbos: Michos, 10 16.5.80 leg. Teunissen, ZT. - Kos: Georgeos-Christos, $10^{*}$ 27.-29.4.71 500m, ZSM. - Libanon: Cedars, 1 o 10.-23.6.62 Sw, $10^{\star}$ coll. SCHMIEDEKNECHT ZMB.

Verbreitung von $A$. asiatica: Ostmediterran. Westtürkei und vorgelagerte griechische Inseln, auch europäischer Teil der Türkei, östlicher und südöstlicher Teil der Türkei (fehlt nach derzeitigem Stand des Wissens im mittleren Teil der Türkei), Libanon (Karte, Fig. 3).

\section{Diskussion}

Durch das Auffinden der vollständigen Typenserie konnten wir anhand des Weibchens aus Eberswalde klären, daß die Nominatform nicht wesentlich dunkler behaart ist als die von WARNCKE beschriebene $A$. a. oxyura und sich auch morphologisch nicht klar von dieser abtrennen läßt: $A$. asiatica oxyura WARNCKE, $1975=$ A. asiatica FrIESE, 1921, syn. $\mathrm{n}$.

Es gibt allerdings einzelne Weibchen (aus Gaybi in der Türkei und aus Cedars im heutigen Libanon), die deutlich dunkler behaart sind als in der obigen Beschreibung angegeben. Da WARNCKE ursprünglich nur die Männchen von A. asiatica kannte (WARNCKE 1965), hat er später (1975) offensichtlich diese dunkel behaarten Weibchen als zur No- 
minatform gehörend gedeutet und dementsprechend die heller behaarten als neue Unterart beschrieben. Die Beschreibung von FRIESE ist in der Tat etwas mißverständlich, was die Haarfarbe anbelangt.

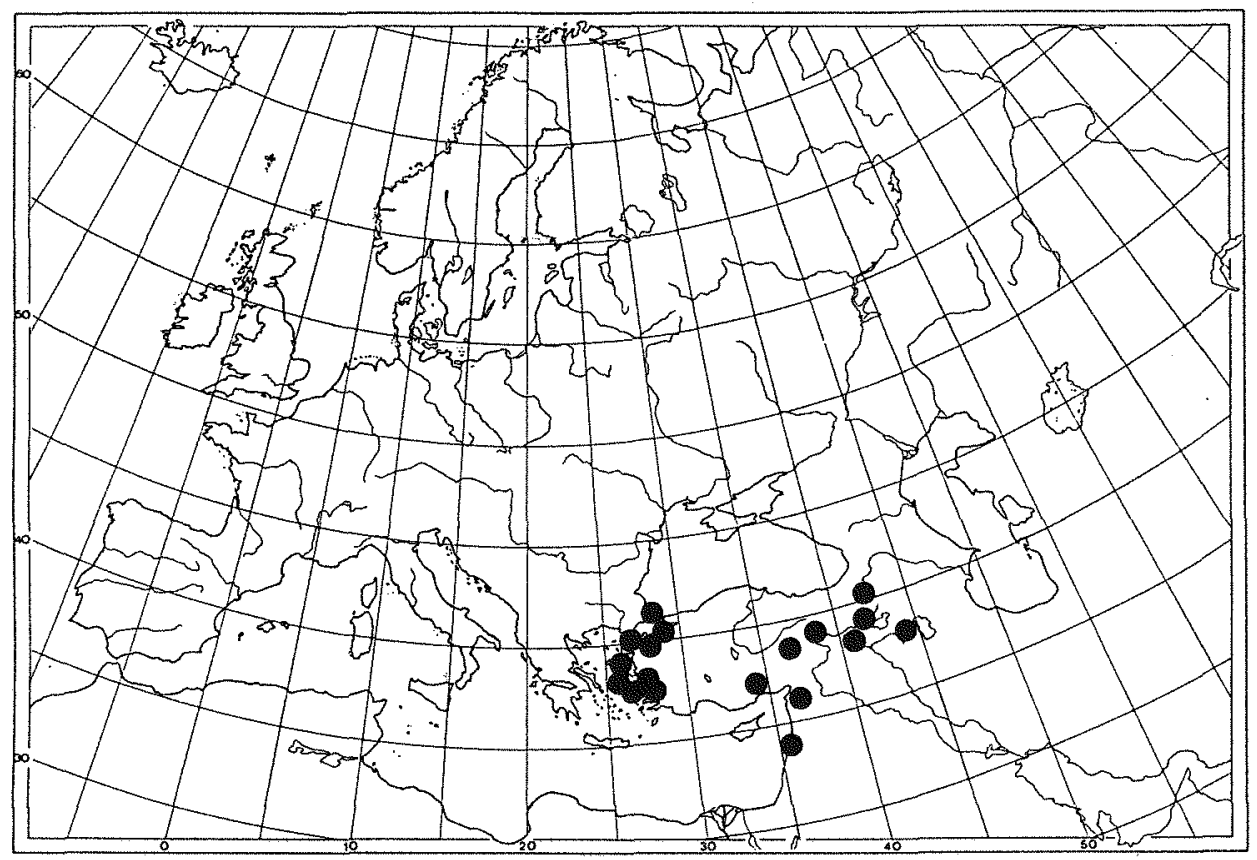

Fig. 3: Bisherige Fundorte ron A. asiatica.

Im Binokular sind bei den dunkel behaarten Weibchen im Vergleich zu den heller behaarten z.T. Unterschiede im Pygidium und im Sensillenmuster der Antennen zu etkennen; für weitergehende REM-Untersuchungen liegt jedoch nicht genug Material vor. Nach den bisherigen Ergebnissen gibt es auch keine weiteren morphologischen Merkmale, die darauf hinweisen, daß es sich bei den heller bzw. dunkel behaarten Tieren um verschiedene Taxa handeln könnte. Viel eher scheint hier eine innerartliche Variation bezüglich der Färbung, z.B. auf Grund verschiedener Temperaturen bei der Entwicklung, vorzuliegen.

Gruppenzugehörigkeit: Nach unseren Untersuchungen gehören folgende Arten zur Andrena nobilis-Gruppe ( $=$ Nobandrena): A. nobilis MORAWTTZ, 1873, A. anatolica AlFKEN, 1935, A. atbenensis WARNCKe, 1965, A. compta LePELETIER, 1841, A. flavobila WARNCKE, 1965, A. fratercula WARNCKE, 1975, A. funerea WARNCKE, 1967, A. probata WARNCKE, 1973 und $A$. acutilabris MORAWITZ, 1876 (cf. SCHÖNITZER et al. 1994). Darüber hinaus wurde jüngst $A$. (Nobandrena) comptaeformis GUSENLEITNER \& SCHWARZ, 2000 beschrieben. Es sprechen eine Reihe von Gründen dafür, daß $A$. asiatica nicht zur A. nobilis-Gruppe gehört. A. asiatica unterscheidet sich von den oben genannten Arten insbesondere in der Ausprägung folgender Merkmale:

(i) die auffällige Behaarung am Thoraxrücken, die an $A$. curvungula erinnert, (ii) die breite Fovea facialis, (iii) die unregelmäßige Skulptur des Mittelfelds des Mittelsegmentes, (iv) die lang gestreckte, vorne relativ spitze Galea, die glänzend und eingestochen punktiert 
ist, (v) die schwarz- bis rötlichbraune Scopa mit rundherum gefiederten Haaren, (vi) die Form der Spitze der Penisvalve. Außerdem (vii) besucht diese Art im Gegensatz zu den Arten $\operatorname{der}$ A. nobilis-Gruppe keine Cruciferen, sondern Campanula.

A. asiatica dürfte mit $A$. formosa MORAWITZ, 1878, $A$. combusta MORAWITZ, 1876 und weiteren Arten verwandt sein. Dafür sprechen insbesondere die Form der Fovea facialis und die Rückenbehaarung.

Anmerkung zur Nomenklatur: $A$. formosa und $A$. combusta wurden von WARNCKE (1967, S. 272 und S. 262) mit A. oulskii RADOSZKOWSKI, 1867 synonymisiert. Es handelt sich aber um jeweils eigenständige Arten: Species revocatae. Begründung: Bei dem von WARNCKE (1967, S. 178) als Typus von A. oulskii RAD., 1867 gedeuteten Weibchen in Betlin handelt es sich in Wirklichkeit um A. combusta MORAwITZ, 1876; die Herkunft dieses Tieres (Afghanistan) stimmt nicht mit dem locus typicus (Kaukasus) überein (es wurde von RADOSZKOWSKI selbst als $A$. oulskiz fehlbestimmt). Der Typus der echten A. oulskii RAD., 1867 wurde von W. GRÜNWALDT im Natural History Museum London gefunden.

\section{Dank}

Für die großzügige und geduldige Ausleihe ron Material danken wir den Herren Dr. A. TAEGER und Dipl.-Biol. S. M. Blank (Eberswalde), Mag. F. GusenleItNer (Linz) und Dr. F. KOCH (Berlin). Herr Dr. R. MELZER (München) ermöglichte uns die Benutzung des Rasterelektronenmikroskopes des Zoologischen Institutes det LMU.

\section{Literatur}

BLANK, S. M. \& KraUS, M. 1994: The nominal taxa described by K. WARNCKE and their types (Insecta, Hymenoptera, Apoidea). - Linzer biol. Beitr. 26: 665-761.

FAHRINGER, J. \& FRIESE, H. 1921: Eine Hymenopteren-Ausbeute aus dem Amanusgebirge (Kleinasien und Nord-Sytien, südl. Armenien). - Arch. Naturg. 87A, 3: 150-180.

GUSENLEITNER, F. 1984: Faunistische und morphologische Angaben zu bemerkenswerten Andrena-Arten aus Österreich. - Linzer biol. Beitr. 16/2: 211-276.

GUSENLEITNER, F. \& SCHWARZ, M. 2000: Nomenklatorische Aktualisierungen in der Bienengattung Andrena sowie Beschreibung einer neuen Art (Hymenoptera: Apidae: Andreninae) - Entomofauna 21 (10): 105-116.

SCHÖNTZER, K.; SCHUBERTH, J. \& GRÜNWALDT, W. 1994: Untersuchungen zur Phylogenie innerhalb der Gattung Andrena. In: HENTSCHEL, E. J. (Hrsg.). 2. Jenaer Bienenkundliche Symposium der FriedrichSchiller-Universität. - Proceedings, Jena 1993 [1994] S. 15.

WARNCKE, K. 1965: Beitrag zur Kenntnis der Bienengattung Andrena F. in der Türkei (Hymenoptera, Apoidea). - Mitt. Münchner Ent. Ges. 55: 244-273.

WARNCKE, K. 1967: Beitrag zur Klärung paläarktischer Andrena-Arten (Hym. Apidae). - EOS 43 (1-2) 171-318.

WARNCKe, K. 1974: Die Sandbienen der Türkei (Hymenoptera, Apoidea, Andrena) Teil A. - Mitt. Münchner Ent. Ges. 64: 8-116.

WARNCKE, K. 1975: Die Sandbienen der Türkei (Hymenoptera, Apoidea, Andrena) Teil B. - Mitt. Münchner Ent. Ges. 65: 29-102.

\footnotetext{
Anschriften der Verfasser:

Dipl.-Biol. JOHANNES SCHUBERTH

und Prof. Dr. KLAUS SCHÖNITZER

Zoologische Staatssammlung München

Münchhausenstraße 21

D - 81247 München
}

Dr. WILHELN GRÜNWALDT

Walterstraße 19

D - 80337 München 